\title{
Oriented crystallization of GaSb on a patterned, amorphous Si substrate
}

\author{
S. S. Yi and P. D. Moran \\ Department of Chemical Engineering, University of Wisconsin, Madison, Wisconsin 53706 \\ $X$. Zhang and F. Cerrina \\ Department of Electrical and Computer Engineering, University of Wisconsin, Madison, Wisconsin 53706 \\ J. Carter and H. I. Smith \\ Department of Electrical Engineering and Computer Science, Massachusetts Institute of Technology, \\ Cambridge, Massachusetts, 02139 \\ T. F. Kuech ${ }^{\mathrm{a})}$ \\ Department of Chemical Engineering, University of Wisconsin, Madison, Wisconsin 53706
}

(Received 4 January 2000; accepted for publication 3 January 2001)

\begin{abstract}
Oriented crystallization of $\mathrm{GaSb}$ on patterned, oxidized Si substrates was achieved by metalorganic chemical vapor deposition. The Si substrate was formed by patterning an array of inverted square pyramids having $\{111\}$ sidewall facets, using lithography and anisotropic etching in $\mathrm{KOH}$. The orientation and structure of GaSb crystals, at various stages of the growth, were examined by scanning electron microscopy and x-ray diffraction. X-ray diffraction pole figure analysis shows that $\{111\}$ planes of GaSb are predominantly parallel to the $\{111\}$ planes of the inverted pyramids. Extra (111) spots observed in the x-ray diffraction pole figure are interpreted in terms of multiple twinning of GaSb. 이 2001 American Institute of Physics. [DOI: 10.1063/1.1352657]
\end{abstract}

Graphoepitaxy refers to the use of surface patterning to achieve orientation in overlayer films. ${ }^{1}$ Several mechanisms of producing orientation via patterning have been described, ranging from induced nucleation, attachment of mobile crystallites, ${ }^{2}$ and guided recrystallization. ${ }^{3}$ Graphoepitaxy may have important application in the preparation of multilayer microstructures where single crystalline films of active materials with different properties are separated by amorphous, passive intermediate layers. The major problem in graphoepitaxy is the achievement of crystallographic perfection comparable to that achieved by conventional epitaxial methods.

GaSb-based compound semiconductors are of great interest for infrared optoelectronic devices over the spectral range from $1.24 \mu \mathrm{m}$ for $\mathrm{AlGaAsSb}$ to $4.4 \mu \mathrm{m}$ for GaInAsSb, as well as for high-speed electronic devices. ${ }^{4}$ Since a semiinsulating substrate is not available in GaSb, semi-insulating GaAs is normally used for electronic applications. A principal concern in the growth of GaSb on GaAs substrates is the large, $8 \%$ lattice mismatch, which leads to a high density of threading dislocations. The dislocation density can be reduced by growing an appropriate thin, low temperature $\mathrm{GaSb}$ buffer layer. ${ }^{5,6}$ However, the optimal growth condition for such an initial buffer layer has not yet been well established. Graphoepitaxy of GaSb may provide an alternative way of growing GaSb films on insulating materials such as $\mathrm{SiO}_{2}$. In this letter, we present an investigation of graphoepitaxy of $\mathrm{GaSb}$ on a patterned, amorphous substrate by metalorganic chemical vapor deposition.

$\mathrm{GaSb}$ films were grown in a vertical quartz reactor operated at 76 Torr. A graphite susceptor was heated by a rf

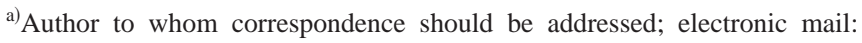
kuech@engr.wisc.edu
}

coil, to a growth temperature of $550-650^{\circ} \mathrm{C}$. Trimethylgallium (TMGa) and trimethylantimony (TMSb) in a $\mathrm{Pd}$ diffused $\mathrm{H}_{2}$ carrier gas were used as $\mathrm{Ga}$ and $\mathrm{Sb}$ precursors, respectively. TMGa and TMSb mole fractions within the reactor were $3 \times 10^{-4}$ and $4 \times 10^{-4}$, respectively.

Silicon (100) substrates were patterned using electron beam lithography and anisotropic etching in $\mathrm{KOH}$ to form a hexagonal-close-packed array of inverted square pyramids, measuring $10 \times 10 \mu \mathrm{m}$ at the base, on $16 \mu \mathrm{m}$ centers. The $\{111\}$ facets of the inverted pyramids are close to atomically smooth. After patterning, the Si substrates were degreased in trichloroethylene, acetone, and isopropanol, and rinsed in deionized (DI) water. To eliminate carbon contaminants on the surface, the substrates were boiled in $\mathrm{HNO}_{3}$ for $10 \mathrm{~min}$, followed by a dip in HF for $15 \mathrm{~s}$, and a rinse in DI water. The substrates were then oxidized in a boiling solution of $\mathrm{HCl}: \mathrm{H}_{2} \mathrm{O}_{2}: \mathrm{H}_{2} \mathrm{O}$ (3:1:1) for 10 min to form a thin amorphous $\mathrm{SiO}_{2}$ film $(\sim 5-8 \AA)$ on the surface. ${ }^{7}$ Since the $\mathrm{SiO}_{2}$ layer is completely amorphous, the crystal structure of underlying $\mathrm{Si}$ is not seen by the growing GaSb layer. After rinsing in DI water, the substrates were blown dry with $\mathrm{N}_{2}$. The grown layers were characterized by Nomarski differential interference contrast microscopy, scanning electron microscopy (SEM), and x-ray diffraction (XRD).

Figure 1 is a SEM micrograph of GaSb grown at $600{ }^{\circ} \mathrm{C}$ on a patterned $\mathrm{Si}$ substrate for $15 \mathrm{~min}$. In the early stage of growth, the film consists of discrete crystals. Preferential nucleation of $\mathrm{GaSb}$ crystallites at the apexes and the corners of inverted square pyramids is evident. However, there is also some nucleation on plateaus and facets. Decreasing the gas-phase supersaturation by lowering the growth temperature to $550^{\circ} \mathrm{C}$ did not greatly reduce nucleation on plateaus. Growth at $650{ }^{\circ} \mathrm{C}$ resulted in many of the inverted square pyramids remaining empty. The growth temperature is close to the melting point of $\mathrm{GaSb}\left(712^{\circ} \mathrm{C}\right)$ and rapid surface dif- 


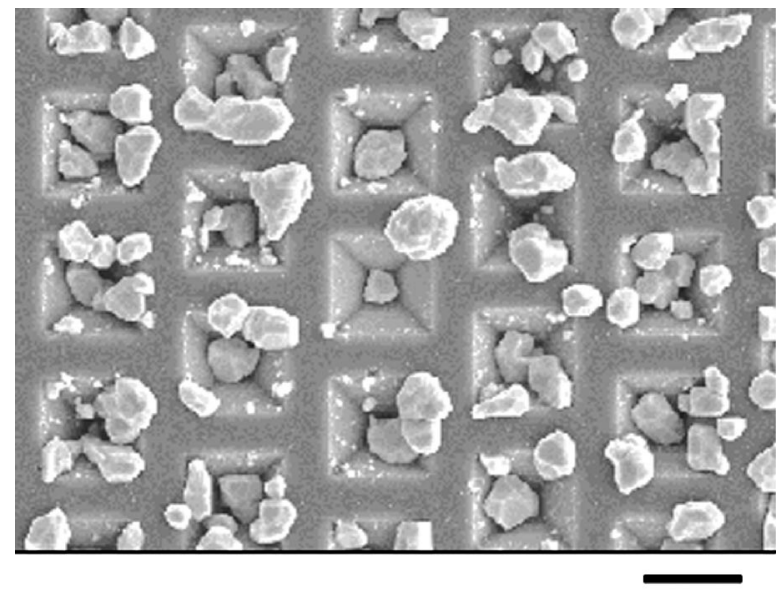

$10 \mu \mathrm{m}$

FIG. 1. SEM micrograph of $\mathrm{GaSb}$ grown at $600{ }^{\circ} \mathrm{C}$ for $15 \mathrm{~min}$ on a patterned, oxidized Si substrate. Preferential nucleation of GaSb crystallites at the apexes and the corners of inverted square pyramids is evident.

fusion could lead to the formation of a few crystallites being fed by a large surface region.

As the growth proceeds, large-grained GaSb crystals grow out of inverted square pyramids. Figure 2 is a SEM micrograph of the sample grown at $600{ }^{\circ} \mathrm{C}$ for $60 \mathrm{~min}$. Electron probe $\mathrm{x}$-ray microanalysis indicates that these largegrained crystals are composed of $50 \% \mathrm{Ga}$ and $50 \% \mathrm{Sb}$. Double-crystal (004) XRD exhibits a strong diffraction peak with full width half maximum (FWHM) of $\sim 1400$ arc sec, indicating that a (100) plane of $\mathrm{GaSb}$ is parallel to the $\mathrm{Si}$ (100) substrate. This FWHM is comparable to that from $\mathrm{GaSb}$ grown on a GaAs substrate without an initial buffer layer. ${ }^{8}$

Figure 3 is an XRD pole figure using the (111) diffraction. There are eight high intensity, evenly spaced spots: four spots at $\sim 55^{\circ}$ and four spots at $\sim 16^{\circ}$ from the surface normal. Since the angle between the $\{100\}$ and $\{111\}$ crystallographic planes is $54.7^{\circ}$ in cubic crystals such as $\mathrm{Si}$ and $\mathrm{GaSb}$, this result indicates that GaSb crystals grown on the patterned $\mathrm{Si}$ substrate have $\mathrm{GaSb}\{100\}$ planes parallel to the $\mathrm{Si}$ $\{100\}$ planes and $\mathrm{GaSb}\{111\}$ planes parallel to $\mathrm{Si}\{111\}$

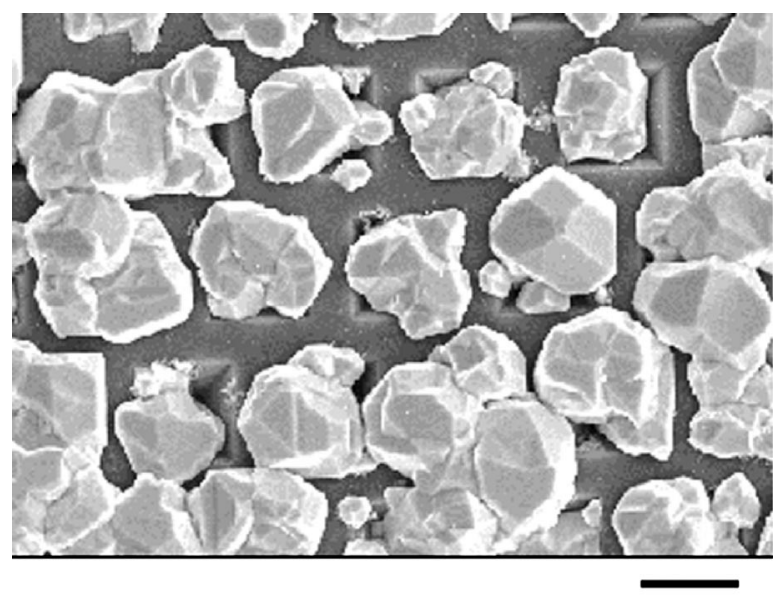

$10 \mu \mathbf{m}$

FIG. 2. SEM micrograph of a GaSb film grown at $600^{\circ} \mathrm{C}$ for $60 \mathrm{~min}$ on a

Four octahedrons are joined to the $\{111\}$ faces of a primary octahedron.
patterned, oxidized Si substrate.

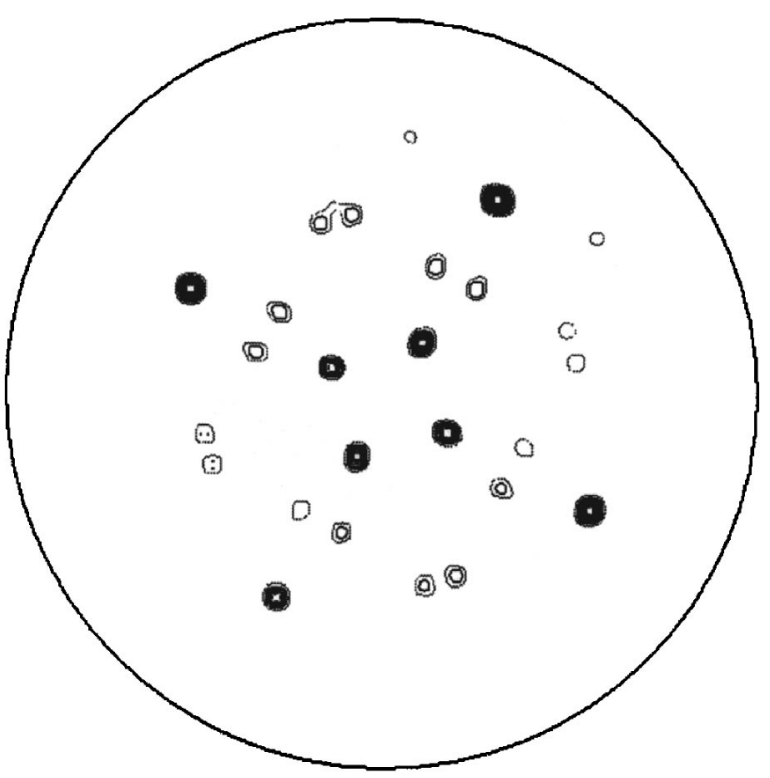

FIG. 3. (111) XRD pole figure of a GaSb film grown at $600{ }^{\circ} \mathrm{C}$ for $60 \mathrm{~min}$.

planes, i.e., epitaxy. In addition, there are four pairs of spots at $\sim 34^{\circ}$, and four pairs of spots at $\sim 45^{\circ}$. The diffraction intensity of these spots are $\sim 4$ times weaker that that of the eight spots. The XRD pole figure measured from the film grown at $550^{\circ} \mathrm{C}$ was found to be identical to that from the film grown at $600^{\circ} \mathrm{C}$.

Multiple twinning of $\mathrm{GaSb}$ is most likely responsible for the extra spots in the pole figure. Ino ${ }^{9}$ used a multiply twinned particle model to explain 24 abnormal (111) spots observed in electron diffraction patterns in the gold films evaporated onto cleaved halide substrates. Twinning in $\mathrm{GaSb}$ crystals is commonly reported in liquid encapsulated Czochraski growth and is sensitive to the direction of growth and the melt composition. ${ }^{10-12}$ Figure 4 is a schematic of multiply twinned $\mathrm{GaSb}$ particles, which are composed of five ideal octahedrons. In this structure, four octahedrons are joined to the four $\{111\}$ faces of a primary octahedron. The primary octahedron is assumed to be congruent with the inverted square pyramids in the patterned Si substrate. Considering that the angle between a (111) plane in a secondary octahedron (marked as S in Fig. 4) and a (100) substrate plane is $15.8^{\circ}$, four $\{111\}$ planes in secondary octahedrons are presumably responsible for the four spots observed at $\sim 16^{\circ}$. Although the origin of eight pairs of spots, four at $\sim 34^{\circ}$ and four at $\sim 45^{\circ}$, is not fully understood at this time, it is likely that different multiple twins having multiple positions on the substrate contribute to the extra pairs of (111) spots observed in the pole figure.
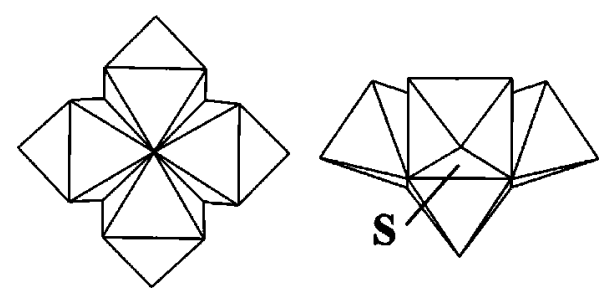

FIG. 4. Top and side schematic view of multiply twinned GaSb particles. Four octahedrons are joined to the $\{111\}$ faces of a primary octahedron. AIP license or copyright, see http://apl.aip.org/apl/copyright.jsp 
It is important to determine whether the orientation observed could be due to growth through pinholes present in the thin $\mathrm{SiO}_{2}$ grown over the $\mathrm{Si}$ substrate. The same question applied to the experiments on GaAs growth over sawtoothpatterned $\mathrm{Si}$, in which case the issue was left unresolved. ${ }^{13}$ Although growth through pinholes cannot be ruled out absolutely, it is noteworthy that GaSb films were grown on plain (100) and (111) $\mathrm{Si}$ substrates covered with $\mathrm{SiO}_{2}$ films prepared in the same way as on the patterned substrate. XRD pole figure analysis on these samples did not show any (111) texture, indicating that pinholes are probably not responsible for the oriented crystallization observed in this study.

A variety of mechanisms can induce oriented crystallization in graphoepitaxy. In the growth of $\mathrm{ZnS}$ on a patterned amorphous (111) Si substrate, the mobility of $\mathrm{ZnS}$ crystallites on the substrate was found to be critical in producing oriented crystallization. ${ }^{3}$ In this case, an ultrathin $(\leqslant 100 \AA)$ $\mathrm{Cu}$ film and a thin $(1-2 \mu \mathrm{m}) \mathrm{CdS}$ film were evaporated onto the substrate to enhance the mobility of $\mathrm{ZnS}$ crystallites since $\mathrm{CdS}$ and $\mathrm{ZnS}$ form eutectic solutions with $\mathrm{Cu}$ that are liquid at temperatures higher than $700-800{ }^{\circ} \mathrm{C}$. Assuming that GaSb nuclei on the $\mathrm{SiO}_{2}$ surface are not highly mobile to facilitate oriented crystallization, the question becomes one of whether orientation occurs at nucleation or during film growth. To obtain more information on the mechanism of oriented crystallization, a GaSb film was grown on a patterned $\mathrm{Si}$ substrate consisting of smaller inverted square pyramids, measuring $0.1 \times 0.1 \mu \mathrm{m}$ at the base, on $0.2 \mu \mathrm{m}$ centers. Interestingly, the film grown on a smaller pattern did not show any (111) texture. This result suggests that there is an interplay of the surface diffusion length, the pattern size, and the local supersaturation for oriented crystallization. Systematic investigation of oriented crystallization as a function of the pattern size would reveal the role of surface supersaturation, diffusion, and surface energy.

This work was supported by the DARPA program on crystal growth (W. S. Coblenz). Facilities support by the NSF UW-MRSEC is gratefully acknowledged.

${ }^{1}$ H. I. Smith, M. W. Geis, C. V. Thompson, and H. A. Atwater, J. Cryst. Growth 63, 527 (1983).

${ }^{2}$ J. D. Filby and S. Nielsen, Br. J. Appl. Phys. 18, 1357 (1967).

${ }^{3}$ E. I. Givargizov, Thin Solid Films 189, 389 (1990).

${ }^{4}$ P. S. Dutta, H. L. Bhat, and V. Kumar, J. Appl. Phys. 81, 5821 (1997).

${ }^{5}$ R. M. Graham, A. C. Jones, N. J. Mason, S. Rushworth, L. Smith, and P. J. Walker, J. Cryst. Growth 145, 363 (1994).

${ }^{6}$ F. Royo, A. Giani, F. Pascal-Delannoy, L. Gouskov, J. P. Malzac, and J. Camassel, Mater. Sci. Eng. B 28, 169 (1994).

${ }^{7}$ A. Ishizaka and Y. Shiraki, J. Electrochem. Soc. 133, 666 (1986).

${ }^{8}$ K. Hjelt and T. Tuomi, J. Cryst. Growth 170, 794 (1997).

${ }^{9}$ S. Ino, J. Phys. Soc. Jpn. 21, 346 (1966).

${ }^{10}$ I. Hirai and T. Obokata, Jpn. J. Appl. Phys., Part 1 21, 956 (1982).

${ }^{11}$ A. Katsui and C. Uemura, Jpn. J. Appl. Phys., Part 1 21, 1106 (1982).

${ }^{12}$ F. Moravec, V. Sestakova, B. Stepanek, and V. Charvat, Cryst. Res. Technol. 24, 275 (1989).

${ }^{13}$ K. Ismail, F. Legoues, N. H. Karam, J. Carter, and H. I. Smith, Appl. Phys. Lett. 59, 2418 (1991). 